\title{
Modelos y aserción empírica: el modelo de doble hélice para la concepción semántica de la ciencia*
}

\author{
Models and Empirical Claim: the Double Helix Model for the Semantic View of
}

Science

Maria de las Mercedes O’Lery ${ }^{\ddagger \ddagger}$

\begin{abstract}
Resumen
La propuesta del presente artículo es poner en comparación el modo de entender los modelos y la relación de éstos con el mundo para dos versiones dentro de la familia semanticista como lo son el realismo constructivo y la concepción estructuralista de las teorías científicas. Para exponer las particularidades de estos dos enfoques, se considerará el caso particular del denominado modelo de doble hélice propuesto por primera vez por James D. Watson y Francis H. C. Crick (Watson \& Crick 1953a). El objetivo de este trabajo es modesto en la medida en que simplemente se intenta subrayar las diferencias entre las dos concepciones antes mencionadas a partir de considerar el tratamiento que cada uno de estos enfoques hace del modelo de doble hélice y, en el mejor de los casos, posibilitar un entendimiento más preciso de los elementos que cada uno de ellos proponen para el análisis de las teorías.
\end{abstract}

Palabras clave: modelos - realismo constructivo - concepción estructuralista - modelo de doble hélice

\begin{abstract}
The proposal in this article is to compare the way of understanding models and their relationship with the world for two approaches within the semantic family such as the constructive realism and the structuralist view of scientific theories. In order to expose the particularities of these two approaches, we will consider the particular case of the so-called double helix model first proposed by James D. Watson y Francis H. C. Crick (Watson \& Crick 1953a). The aim of this work is modest in that it is simply attempted to emphasize the differences between the two above mentioned approaches by considering the treatment that each of these approaches makes of the double helix model and, at best, to enable a more precise understanding of the elements that each propose for the analysis of theories.
\end{abstract}

Keywords: models - constructive realism - structuralist view - double helix model

\footnotetext{
* Recibido: 27 de diciembre de 2015. Aceptado con revisiones: 14 de marzo de 2016.

+ Universidad Nacional de Quilmes y Universidad de Buenos Aires-CBC, Argentina. Para contactar a la autora, por favor, escribir a: mercedesolery@yahoo.com.ar.

* Este trabajo ha sido realizado con la ayuda de los proyectos de investigación PICT2012-2662 (ANPCyT, Argentina), PICT- 2014- 1741 (UNQ, Argentina) y UBACyT 2014-2017/Código: 20020130100814BA (UBA, Argentina). Agradezco a Daniel Blanco y Santiago Ginnobilli por los valiosos comentarios, sugerencias y correcciones efectuadas a la primera versión de este trabajo. Metatheoria 7(1)(2016): 17-30. ISSN 1853-2322. eISSN 1853-2330.

(c) Editorial de la Universidad Nacional de Tres de Febrero. Publicado en la República Argentina.
} 


\section{Introducción}

La pregunta filosófica, tanto en lo que respecta al conocimiento en general como al conocimiento científico en particular, acerca de cómo y cuán relacionadas están nuestras afirmaciones acerca del mundo con dicho mundo ha sido, y es aún hoy, objeto de persistente análisis.

En lo estrictamente referente al conocimiento científico, los elementos que permitirían dar cuenta de las relaciones establecidas entre las teorías y el mundo para la así denominada concepción clásica, heredada o sintáctica de la ciencia son las llamadas reglas de correspondencia. Éstas constituyen un elemento presente en la elucidación del concepto de teoría empírica propuesta por los autores pertenecientes a dicha tradición. Tomando el caso de Rudolf Carnap, uno de los principales representantes de la concepción clásica, una teoría consiste en un conjunto de enunciados organizados deductiva o axiomáticamente. En el caso de las teorías empíricas, tal conjunto se presenta bajo la forma de un sistema "interpretado" cuyos componentes son: (a) un cálculo específico a partir del cual se enuncian los axiomas o leyes de la teoría; y (b) reglas semánticas a partir de las cuales se hace posible una interpretación para dicho cálculo. Cuando se trate de una teoría empírica, tal interpretación se hará necesaria dado que debe existir algún tipo de relación entre lo afirmado por la teoría y las situaciones reales concretas. El papel designado a las reglas de correspondencia es el de posibilitar tal interpretación. Las reglas de correspondencia son reglas semánticas, son enunciados en los que se establece cierto vínculo entre al menos uno de los términos teóricos propuestos por la teoría y al menos uno de sus términos descriptivos (Diez \& Lorenzano 2002, pp. 14-21).

La cuestión de la vinculación de los conceptos teóricos con la experiencia planteada por la concepción clásica, así como otras de no menor relevancia, ha sido objeto de revisión en las últimas décadas, entre otros, por parte de la así llamada concepción modelo-teórica o semántica de la ciencia. Tal como ocurre en el caso de la concepción clásica donde el consenso acerca de concebir una teoría científica como una entidad lingüística, específicamente como una clase de enunciados organizados axiomáticamente, no excluye la posibilidad del disenso por parte de los autores de tal tradición acerca de otras cuestiones, la concepción semántica o modelo teórica se manifiesta a partir de una familia de concepciones que, más allá de las diferencias, coinciden en apelar a clases de modelos para identificar a las teorías, y no a clases de enunciados. En su acepción informal, un modelo, aun cuando los partidarios de la concepción modelo-teórica mantengan diferencias específicas en el modo de entender tales entidades, consiste en un sistema o estructura que pretende representar de algún modo un "trozo de la realidad”. Los modelos no son entidades lingüísticas pero sí se determinan a partir de tales entidades. Son los axiomas, leyes o principios de la teoría los que permiten definir los modelos. Éstos, así definidos, realizan afirmaciones acerca del modo en que se comportan los sistemas "físicos" o "empíricos" concretos, es decir, las entidades del mundo a las que pretenden representar o capturar. La relación afirmada entre los sistemas empíricos de los que se quiere dar cuenta y los modelos que se presentan para tal fin se expresa a partir de un enunciado, la afirmación o aserción "empírica" de la teoría. Dependiendo de la versión modelo-teórica que se esté considerando, este enunciado afirmará de qué tipo es la relación establecida entre "teoría” y “experiencia” (Diez \& Lorenzano 2002, p. 34).

La propuesta del presente trabajo es poner en comparación el modo de entender los modelos y la relación de éstos con el mundo para dos versiones dentro de la familia semanticista o modelo-teórica como lo son el realismo constructivo de Ronald Giere y la concepción estructuralista de las teorías científicas cuyos principales representantes son Joseph D. Sneed, Wolfgang Stegmüller, C. Ulises Moulines y Wolfgang Balzer. Para exponer las particularidades de cada uno de estos dos enfoques semanticistas, se recurrirá al caso particular del denominado modelo de doble hélice propuesto por primera vez por James D. Watson y Francis H. C. Crick (Watson \& Crick 1953a) para describir la estructura de la molécula de ADN. 
El objetivo perseguido es modesto en la medida en que simplemente se intenta, a partir de considerar el tratamiento que cada uno de dichos enfoques hace del modelo de doble hélice, subrayar las diferencias entre ambas concepciones y, en el mejor de los casos, posibilitar a partir del contraste un entendimiento más preciso de los elementos que proponen para el análisis de las teorías.

\section{La estructura del àcido desoxiribonucleico}

Hacia mediados de la década de 1950 se produjo un cambio fundamental en la orientación de la investigación genética. En abril de 1953, se publica en el volumen 171 de Nature el artículo "Molecular Structure of Nucleic Acid: A Structure for Deoxyribose Nucleic Acid”. Allí, James D. Watson y Francis H. C. Crick sugieren que la estructura secundaria de la molécula de ADN presenta la forma de una doble hélice. Para arribar a tal conclusión, Watson y Crick tomaron en cuenta estudios y observaciones llevadas a cabo por otros investigadores. Poco antes de la publicación de Watson y Crick, Linus C. Pauling y Robert Corey, a partir del descubrimiento de la estructura de la $\alpha$-hélice, habían sugerido una estructura en forma de triple hélice para la molécula de ADN. ${ }^{1}$ Watson y Crick también tenían conocimiento acerca de los estudios de Edwin Chargaff sobre la composición y las proporciones de las bases nitrogenadas. ${ }^{2} \mathrm{Y}$, por otro lado, estaban al tanto de los estudios de difracción de rayos-X sobre el ácido desoxiribonucleico realizados de manera independiente tanto por Rosalind E. Franklin y Raymond Gosling como por Maurice H. F. Wilkins; aun cuando, al menos al momento de la publicación del artículo, los consideraban insuficientes para proporcionar una prueba rigurosa de la estructura de doble hélice que proponían. ${ }^{3}$ Paradójicamente, la fotografía del patrón de difracción de cristales de ADN que contribuyó decisivamente a una confirmación del modelo de doble hélice aparece en un artículo de R. Franklin publicado en el mismo número de Nature en el que apareciera el citado artículo de Watson y Crick (Franklin \& Gosling 1953).

De acuerdo con Waston y Crick, la estructura de la molécula de ADN se presenta bajo la forma de dos cadenas helicoidales enrolladas en torno al mismo eje. Cada cadena consiste en grupos fosfatodiéster uniendo residuos de ß-D-desoxirribofuranosa por medio de enlaces 3',5'. Las dos cadenas (pero no sus bases) se relacionan por una díada perpendicular al eje de la fibra. Ambas cadenas siguen una hélice dextrógira, pero debido a las díadas las secuencias de átomos en las dos cadenas corren en direcciones opuestas. Para cada una de las cadenas, las bases están sobre la parte interna de la hélice y los fosfatos en la externa. El aspecto novedoso de la estructura, tal como lo sostenían Watson y Crick, es la manera en que las dos cadenas se mantienen unidas por bases púricas y pirimidínicas reunidas en pares. Una del par debe ser purínica y la otra pirimidínica. La estructura sugerida para el ADN, afirmaban los autores, permitía además explicar los procesos de replicación y mutación del material genético. Esto, sin embargo, lo expusieron en "Genetical Implications of the Structure of Deoxyribonucleic Acid", publicado en el mismo volumen de Nature (Watson \& Crick 1953b). La propuesta de Watson y Crick acerca de la estructura de la molécula de ADN constituye el habitualmente llamado modelo de doble hélice.

A continuación, y tal como se anticipó, se empleará este modelo como un caso particular para ejemplificar las diferencias y/o similitudes entre el enfoque defendido por Ronald Giere y la concepción estructuralista de las teorías. Primeramente se expondrá lo relativo a la consideración de Giere acerca de los modelos, haciendo pié especialmente en su análisis del modelo de doble hélice. Seguidamente, se expondrá un tratamiento de dicho modelo de acuerdo con la concepción

\footnotetext{
${ }^{1}$ La propuesta de Pauling de una triple hélice aparece en un artículo publicado, en colaboración con Robert B. Corey, en el mismo número de Nature en el que aparece el artìculo de Watson y Crick (Pauling \& Corey 1953a, 1953b). Ellos, sin embargo, tuvieron acceso a una versión manuscrita del trabajo de Pauling y Corey antes de su publicación. El modelo propuesto por Pauling consistía en tres cadenas entrelazadas, con los fosfatos cerca del eje y las bases hacia fuera. Watson y Crick, sin embargo, presentaron argumentos para considerar poco satisfactoria una estructura tal (Watson \& Crick 1953a, p. 737).

2 En el trabajo de Watson y Crick se hace referencia explícita a los artículos de Zamenhof, Brawerman \& Chargaff (1952) y Wyatt (1952).

${ }^{3}$ Watson y Crick sólo refieren a tal respecto los artículos de Astbury (1947) y Wilkins \& Randall (1953).
} 
estructuralista de la ciencia. Para esto último, se apelará principalmente a la reconstrucción estructuralista de la genética molecular llevada a cabo por W. Balzer y C. M. Dawe (Balzer \& Dawe 1990).

\section{Realismo constructivo y modelo de doble hélice}

Como es sabido, el así llamado realismo constructivo, propuesto por Ronald Giere, surge paradójicamente aprovechando los desarrollos que van Fraassen presenta para proporcionar una forma lingüística alternativa para las teorías científicas más adecuada al empirismo. ${ }^{4}$ El realismo constructivo se origina como una versión realista alternativa al empirismo constructivo de van Fraseen (Giere 1988, p. 93). Giere afirma que "al liberar al Empirismo de sus grilletes positivistas, sin embargo, involuntariamente, van Fraassen también ha puesto en libertad el realismo que él aborrece" (Giere 1985, p. 75).

Es la concepción defendida por van Fraassen acerca de la naturaleza de las teorías científicas lo que le permite "vivir" tanto al empirismo como al realismo, aun cuando él haya preferido defender sólo la posibilidad del empirismo abocándose primariamente a cuestiones de justificación de las teorías. Así, Giere reconoce su deuda al empirismo constructivo de van Fraassen sintiéndose en la envidiable posición de, como él mismo afirma, emplear sus mejores desarrollos como armas contra sus más débiles defensas (Giere 1985, p. 75).

De acuerdo con van Fraassen, la preocupación del empirismo lógico por la estructura lingüística de las teorías científicas impidió que se reconociera la importancia de los modelos que satisfacen tales estructuras. Giere concuerda con van Fraassen en que, en ciencia, el lugar central lo ocupan los modelos y no las formas lingüísticas; sin embargo, discrepa acerca de la naturaleza de estos modelos. Siguiendo a Patrick Suppes, van Fraassen considera que el lenguaje propio para el estudio filosófico de la ciencia es el matemático. A diferencia de Suppes, sin embargo, para quien el lenguaje canónico debe ser aquel proporcionado por la teoría de conjuntos, van Fraassen prefiere no atenerse a tal restricción. Mientras que para Suppes los modelos son concebidos como estructuras conjuntistas, para van Fraassen los modelos son propuestos como "puntos" o "trayectorias" en un espacio de estados. Esta comparación, aun cuando no será éste el lugar donde se revisarán tales nociones, permite marcar una diferencia con respecto a la naturaleza que Giere atribuye a los modelos. Para Giere, los modelos no se caracterizan ni como entidades conjuntistas a la manera de Suppes, ni a partir de espacios de estado como para van Fraassen. La noción de modelo teórico propuesta por Giere es más bien amplia e imprecisa. Mediante el término "modelo teórico" puede referirse tanto a un modelo general como a una de sus versiones específicas obtenida a partir de especificar valores únicos para todos los parámetros y condiciones iniciales. Un modelo teórico es, en principio, una entidad definida (Giere 1985, p. 78). El modelo describe la conducta de una entidad abstracta definida a partir de las leyes o postulados de la teoría.

Giere entiende los modelos como "herramientas para representar el mundo" (Giere 1999, p. 45). Apela, de este modo, a otros usos de los modelos en ciencia que se sumen a los explicitados por Suppes (1960, pp. 287-301). Para él, los modelos constituyen la principal (aun cuando no la única) herramienta para tal fin. La representatividad de los modelos, sin embargo, debe ser analizada desde un punto de vista pragmático. Es decir, los modelos en sí no representan, son los científicos quienes utilizan ciertos modelos para representar determinados aspectos del mundo teniendo en cuenta propósitos específicos. Representar, entonces, desde un punto de vista pragmático, consiste en una relación n-ádica tal que: "los científicos $(\mathrm{C})$ recurren a modelos $(\mathrm{X})$ para representar aspectos del mundo (M) de acuerdo con propósitos específicos (P)" (Giere 2004, p. 743).

Las teorías no se relacionan directamente con el mundo, sino de manera indirecta a partir de los modelos. Las conexiones entre los modelos (como entidades abstractas) y el mundo (como entidades o

\footnotetext{
${ }^{4}$ De acuerdo con Giere, el término "constructivo" pone el acento en el aspecto pragmático enfatizando el hecho de que los modelos son deliberadamente creados, "socialmente construidos", por los científicos (Giere 1988, p. 93).
} 
sistemas reales) están especificadas por lo que Giere denomina hipótesis teórica. Ésta asocia las descripciones del sistema abstracto (dadas, generalmente, por medio de un conjunto de ecuaciones) con descripciones idealizadas de sistemas reales. Es por medio de tales hipótesis que los modelos (los cuales, como tales, son estructuras abstractas) logran representar sistemas físicos concretos; y, las teorías, adquirir contenido empírico.

Pero, ¿qué tipo de relación se establece entre nuestros modelos teóricos y los sistemas reales? O bien, ¿cuál es la relación entre estas entidades (abstractas y reales) afirmada por la hipótesis teórica? De acuerdo con Giere, si nuestro interés es contar con enunciados científicos que sean realistas y que a la vez tengan alguna oportunidad de ser verdaderos, debemos prevenirnos de afirmar que un sistema real determinado sea capturado exactamente mediante algún modelo. Alternativamente, podría sugerirse, tal como lo defienden muchos realistas, que el sistema real es al menos aproximadamente capturado por el modelo. Esto, sin embargo, exigiría explicar qué significa "aproximadamente". Por lo tanto, la propuesta de Giere consiste en considerar a las hipótesis teóricas como proporcionando un vínculo entre un modelo y el mundo a partir de afirmar que un sistema físico es (o está) muy cercano a la entidad abstracta descripta mediante el modelo. De acuerdo con Giere, la hipótesis teórica deberá respetar la siguiente formulación: "El sistema real designado es similar al modelo propuesto en aspectos específicos y en grados específicos" (Giere 1985, p. 80). Es decir, la relación afirmada por la hipótesis teórica entre un modelo y el mundo es siempre una relación de similaridad. A partir de una hipótesis tal se establece que la conducta de un sistema físico concreto es "similar" a la conducta de la entidad abstracta descrita por el modelo.

En lo que respecta al modelo de doble hélice, es bien sabido que Watson decidió evaluar la posibilidad de que la molécula de ADN tuviera dos cadenas y que las bases se encontraran hacia adentro construyendo modelos de ADN, no modelos teóricos sino modelos a escala usando pedazos de alambre, metal y cartón a fin de poder precisar los supuestos planteados. Este recurso no sólo le sería útil para clarificar sus hipótesis sino que además, algunos años antes, le había permitido invalidar la posibilidad de que el número de cadenas de la molécula fuera tres, algo que había conjeturado en un primer momento junto a Crick, antes de que presentaran el modelo de doble hélice. Para representar las bases nitrogenadas utilizó placas de metal, las cuales primeramente fueron de cartón. Watson fue uniendo estas piezas de modo tal que sus formas coincidieran. Las figuras de cartón que representaban bases purínicas encajaban con las que representaban pirimidínicas, específicamente una guanina con una citosina y una adenina con una timina. Ordenó a su vez estas combinaciones formando una escalera en espiral.

Cuando por modelo de doble hélice se está considerando este esquema físico o maqueta construida por Watson, se está concibiendo a esta representación física consistente en alambre y cartón como un modelo icónico de la estructura del ADN. Un modelo icónico cumple la función de ser "ícono" de aquello que están modelando (Suppe 1977, p. 97). En este caso, la maqueta de Watson es una representación física parcial y a escala de la molécula de ADN.

Para Giere, la importancia de tales modelos a escala radica en que proveen un medio satisfactorio para definir un modelo teórico. En este caso, el modelo teórico es un ácido nucleico idealizado con sub-unidades idealizadas compuestas de átomos individuales idealizados en muy específicas disposiciones espaciales. Así, de acuerdo con Giere, la hipótesis teórica afirmada por Watson fue que la molécula de ADN era muy similar en composición y estructura al modelo teórico, si bien en la práctica remitieron directamente al modelo de escala (Giere 1985, p. 91).

\section{La concepción estructuralista de la ciencia y el modelo de doble hélice}

La concepción estructuralista propone considerar a las teorías a partir del análisis de aquello que plantean como dos componentes esenciales de las mismas. El primero de los componentes es el núcleo (K) desde el cual se expresa la parte "formal" de la teoría. El núcleo contiene una serie de modelos que 
permiten expresar las leyes o postulados de la teoría en cuestión. Un modelo es concebido como una entidad abstracta acerca de la cual tienen sentido las hipótesis de una teoría. La noción de modelo que se está suponiendo es la defendida por Suppes (1981, p. 311 y 1960, p. 109). Un modelo, así entendido, es una entidad conjuntista que satisface los axiomas de la teoría. El segundo componente es el dominio de aplicaciones intencionales o pretendidas (I) a partir del cual se expresan los sistemas reales concretos en los que el científico pone su atención y los que intenta aplicar las leyes de la teoría. Estos sistemas pretendidos, por lo tanto, no son caracterizados en términos abstractos como ocurre en el caso de los modelos del núcleo $(\mathrm{K})$, sino que dependen de un acuerdo o consenso entre los científicos pertenecientes a una misma comunidad científica en un momento histórico particular. El núcleo $(\mathrm{K})$ y el dominio de aplicaciones intencionales (I) representan la parte formal y aplicativa, respectivamente, de un elemento teórico básico. Estos elementos, articulados en niveles de especificidad, son constitutivos de una red teórica desde la cual se hace posible describir la estructura sincrónica de una teoría. Considerando el caso de la genética, por ejemplo, su red teórica supone un primer elemento teórico básico a partir del cual se siguen líneas de refinamiento y especialización. En lo que sigue, se pondrá la atención en sólo uno de tales refinamientos, a saber, aquel que caracteriza a la genética molecular y que es el que permite el tratamiento del modelo de doble hélice. ${ }^{5}$ Se comenzará, sin embargo, presentando el elemento teórico básico propuesto desde la metatheoría estructuralista para la genética $(T=\langle K(G)$, $\mathrm{I}(\mathrm{G})\rangle)$ y, posteriormente, el de la genética molecular $(\mathrm{T}=\langle\mathrm{K}(\mathrm{GM}), \mathrm{I}(\mathrm{GM})\rangle)$ defendido como un refinamiento del primero. ${ }^{6}$

\subsection{Los modelos de la genética}

De acuerdo con el análisis estructuralista, los modelos potenciales para la genética, pueden definirse mediante el siguiente predicado (Lorenzano 2002, pp. 267-272):

\section{Definición 1:}

$\mathbf{M}_{\mathrm{p}}(\mathrm{G}): \quad x=\langle\mathrm{J}, \mathrm{P}, \mathrm{G}, \mathrm{APP}, \mathrm{MAT}, \mathrm{DIST}, \mathrm{DET}, \mathrm{COMB}\rangle$ es una genética potencial $(x=\langle\mathrm{J}, P, \mathrm{G}, \mathrm{APP}$, MAT, DIST, DET, COMB $\left.\rangle \in \mathrm{M}_{\mathrm{p}}(\mathrm{G})\right)$ syss

(1) $J$ es un conjunto finito, no vacío

(2) $P$ es un conjunto finito, no vacío

(3) G es un conjunto finito, no vacío

(4) APP: $J \rightarrow P$

(5) MAT: $J \times J \rightarrow \operatorname{Po}(J)$ es una función parcial

(6) DIST: $P \times P \rightarrow \mathrm{D}(P)$ es una función parcial

(7) Para toda $i, i^{\prime} \in J$ tal que MAT esté definida para $\left\langle i, i^{\prime}\right\rangle$ y para toda $\pi \in P$ : $\operatorname{DIST}\left(\operatorname{APP}(i), \operatorname{APP}\left(i^{\prime}\right)\right)(\pi)=F R\left(\pi / \operatorname{MAT}\left(i, i^{\prime}\right)\right)$

(8) DET: $G \rightarrow P$ es una función sobreyectiva

(9) COMB: $\mathrm{G} \times \mathrm{G} \rightarrow \mathrm{D}(\mathrm{G})$.

Donde:

(1) J representa un conjunto de individuos genéticos, que pueden ser tanto individuos propiamente dichos como poblaciones. Se emplea la variable $i$ para indicar "individuos genéticos".

(2) P representa el conjunto de fenotipos. Se emplea la variable $\pi$ para indicar "fenotipos".

(3) G representa el conjunto de genotipos. Se emplea la variable $\gamma$ para indicar "genotipos".

(4) APPEARANCE ("appearance": APP: $i \rightarrow \pi$ ) le asigna a cada individuo su fenotipo. Las ecuaciones tienen la siguiente forma:

\footnotetext{
${ }^{5}$ Puede consultarse Lorenzano (2002) para una detallada exposición de otro refinamiento de la genética que caracteriza a la teoría del gen junto a sus principales líneas de especialización.

${ }^{6}$ Se expone aquí la reconstrucción estructuralista de la genética llevada a cabo por Pablo Lorenzano (ver Lorenzano 2002, pp. 263-281) y la propuesta de reconstrucción de la genética molecular de Wolfgang Balzer y Chris A. Dawe (ver Balzer \& Dawe 1990, pp. 89-110).
} 
APPEARANCE $\left(\right.$ PARENT $\left._{1}\right)=$ PHENOTYPE $_{1}$ APPEARANCE $\left(\right.$ PARENT $\left._{2}\right)=$ PHENOTYPE $_{2}$ APPEARANCE $\left(\right.$ PROGENY $\left.{ }_{i}\right)=$ PHENOTYPE_OF_PROGENY $($ en donde $i \leq n, j \leq k)$.

(5) MATOR ("mator": $\operatorname{MAT}\left(i, i^{\prime}\right)=\left\langle i_{1}, \ldots, i_{n}\right\rangle$ ) representa la transición de los padres a su descendencia. Le asigna a dos padres cualesquiera su descendencia: MATOR $\left(\right.$ PARENT $_{1}$, PARENT $\left._{2}\right)=\left\langle\right.$ PROGENY $_{1}, \ldots$, PROGENY $\left._{n}\right\rangle$ (en donde $n$ varía de acuerdo a los progenitores).

(6) DISTRIBUTOR (“distributor": $\left.\operatorname{DIST}\left(\pi, \pi^{\prime}\right)=\left\langle r_{1} \pi_{1}, \ldots, r_{k} \pi_{k}\right\rangle\right)$ describe la transición de fenotipos paternos a distribuciones de fenotipos en la descendencia.

(7) DISTRIBUTOR (definición de DISTRIBUTOR en términos de frecuencias relativas) puede ser definido por medio de MATOR y de APPEARANCE.

(8) DETERMINER (“determiner": $\operatorname{DET}(\gamma)=\pi$ ) es una función que asigna fenotipos a los genotipos.

(9) COMBINATOR (“combinator": $\left.\operatorname{COMB}(\gamma, \gamma)=\left\langle\alpha_{1} \gamma_{1}, \ldots, \alpha_{s} \gamma_{s}\right\rangle\right)$ representa la transición de genotipos paternos a genotipos en la descendencia. Constituye una función análoga a DISTRIBUTOR sólo que definida sobre los genotipos.

Hasta aquí, los modelos potenciales de la genética expresan los axiomas impropios de la teoría. Los axiomas propios, por otro lado, aquellos mediante los cuales se expresa la ley fundamental de la genética, estarán dados a partir de la definición de los modelos actuales de la teoría. Estos constituyen un subconjunto de los modelos potenciales para la genética partir de que se presentan como estructuras que, además de satisfacer los axiomas impropios, satisfacen la ley fundamental de coincidencia. ${ }^{7}$ Los modelos actuales para la genética, entonces, son presentados mediante la siguiente definición (Lorenzano 2002, p. 272):

\section{Definición 2:}

$\mathbf{M}(\mathrm{G}): \quad$ si $x=\langle J, P, G, A P P, M A T, D I S T, D E T, C O M B\rangle$ es un $\mathbf{M}_{p}(G)$, entonces $x$ es una genética $(x=$ $\langle J, P, G, A P P, M A T, D I S T, D E T, C O M B\rangle \in \mathrm{M}(\mathrm{G}))$ syss

1) para toda $i, i^{\prime} \in J$ tal que MATOR esté definido para $\left\langle i, i^{\prime}\right\rangle$ y para toda $\gamma, \gamma \in G$ tal que $\operatorname{DET}(\gamma)=$ $\operatorname{APP}(i)$ y $\operatorname{DET}\left(\gamma^{\prime}\right)=\operatorname{APP}\left(i^{\prime}\right)$ :

$$
\operatorname{COMB}\left(\gamma, \gamma^{\prime}\right)=\operatorname{DIST}\left(\operatorname{DET}(\gamma), \operatorname{DET}\left(\gamma^{\prime}\right)\right) \text {. }
$$

De acuerdo con la metateoría estructuralista, además de los modelos potenciales y los actuales, en el núcleo teórico básico de una teoría se encuentra una clase de modelos cuya importancia está asociada a la posibilidad de precisar las aplicaciones pretendidas o intencionales de una teoría. Los así llamados modelos potenciales parciales son aquellos cuya estructura posee todos los componentes de la estructura de los modelos potenciales excepto los componentes teóricos. En lo que respecta al caso de la genética, sus modelos potenciales parciales quedan definidos de la siguiente manera: ${ }^{8}$

\footnotetext{
${ }^{7}$ El uso de las herramientas estructuralistas permite hacer explícita la ley fundamental de la genética. En la literatura genética no se encuentra expresada una ley tal, sin embargo, el axioma de coincidencia puede defenderse como expresando una ley fundamental, es decir, una ley válida para todos los modelos de la genética a partir de que cumple los requisitos exigidos a una ley tal. Al respecto, ver Lorenzano (2002, p. 272), y Lorenzano (2005).

${ }^{8}$ La tarea de definir los modelos parciales para una teoría supone previamente identificar los términos teóricos para dicha teoría. Para el estructuralismo, el carácter teórico de un término se determina siempre en relación a, o en el marco de, la teoría en la que se hace presente tal término. De allí que el criterio sea uno de T-teoricidad, es decir, un término será considerado teórico o no-teórico para una teoría, aquella que esté en discusión. De acuerdo con tal criterio, los términos teóricos "GENOTYPE", "DETERMINER" y "COMBINATOR" son defendidos como T-teóricos para la genética. De ahí que no aparezcan en la estructura de los modelos parciales. No será expuesto aquí el criterio de T-teoricidad, para tal fin puede consultarse Balzer, Moulines \& Sneed (1987, pp. 62-78). Para la evaluación y la defensa acerca de la G-teoricidad (teoricidad respecto de la genética) de "GENOTYPE", "DETERMINER" y “COMBINATOR”, ver Lorenzano (2002, pp. 276-278).
} 


\section{Definición 3:}

$\mathbf{M}_{\mathrm{pp}}(\mathrm{G}): \quad y=\langle J, P, A P P$, MAT, DIST $\rangle$ es una genética parcial $\left(y=\langle J, P\right.$, APP, MAT, DIST $\left.\rangle \in \mathbf{M}_{\mathrm{pp}}(\mathrm{G})\right)$ syss existe una $x$ tal que

1) $x=\langle J, P, G, A P P, M A T, D I S T, D E T, C O M B\rangle \in \mathbf{M}_{p}(G)$

2) $y=\langle J, P, A P P, M A T, D I S T\rangle$.

Como se indicó anteriormente, las aplicaciones intencionales, junto con el núcleo teórico básico, constituyen el par de componentes que forman el elemento teórico básico de una teoría. Los "trozos" del mundo, los sistemas empíricos concretos, acerca de los cuales una teoría pretende dar cuenta no pueden ser identificados a partir de aspectos puramente formales, pues son los científicos los que deciden a qué fenómenos desean aplicar su teoría. Aun así, formalmente puede afirmarse que el dominio de aplicaciones intencionales (I) es un subconjunto de los modelos parciales de una teoría. Es decir, una aplicación pretendida para una teoría en especial es un modelo parcial que además es considerado por una comunidad científica determinada como uno de los fenómenos acerca de los cuales la teoría en cuestión quiere dar cuenta.

A partir de lo anterior, puede ahora presentarse lo relativo a la aserción empírica para una teoría. De acuerdo con el enfoque estructuralista, mediante la aserción empírica se afirma una relación entre los modelos de la teoría y los sistemas empíricos concretos acerca de los cuales se pretende dar cuenta. Análogamente a la hipótesis teórica de Giere, la aserción empírica es un enunciado que afirma una relación entre "teoría” y "experiencia”. Un elemento teórico $\mathrm{T}=\langle\mathrm{K}, \mathrm{I}\rangle$ hace posible la formulación de aserciones empíricas. Para formular la aserción empírica asociada a un elemento teórico, la concepción estructuralista se sirve de la noción de contenido de un elemento teórico (Balzer, Moulines \& Sneed 1987, pp. 89-92).

Este contenido es el conjunto de la clase de modelos potenciales parciales que sean compatibles con aquellos componentes del núcleo teórico que impongan cierto tipo de restricción (la/s ley/es, las condiciones de ligadura y los vínculos interteóricos). ${ }^{9}$

Un modo preciso de formular la aserción empírica asociada con un elemento teórico dado $\mathrm{T}=\langle\mathrm{K}$, I $\rangle$, puede ser éste: todas las aplicaciones intencionales pueden ser extendidas con términos teóricos tales que las estructuras resultantes sean modelos potenciales que además estén sometidos a ciertas restricciones (ley/es, condiciones de ligadura y vínculos interteóricos). De este modo, lo que se está afirmando mediante la aserción empírica es que el dominio de aplicaciones intencionales es un miembro del contenido de la teoría. Formalmente, puede expresarse como $\mathrm{I} \in \mathrm{Cn}(\mathrm{K})$, donde " $\mathrm{Cn}(\mathrm{K})$ " expresa el contenido de una teoría, es decir, la clase de modelos potenciales parciales respecto de los cuales $\mathrm{T}$ puede aplicarse exitosamente. En el caso de la genética, la aserción empírica quedaría expresada de la siguiente manera:

Para todo par parental dado que se cruce y produzca descendencia, las distribuciones genéticas de genotipos - producidas por COMBINATOR-y de fenotipos -dadas por DISTRIBUTOR- en la descendencia de ese par coincidirán idealmente -a partir de DETERMINER- las unas con las otras. (Lorenzano 2002, p. 273)

Puesto que el dominio de aplicaciones intencionales de una teoría se identifica a partir de aspectos no formales, independientemente del núcleo teórico de la teoría, para contrastar la afirmación de que $\mathbf{I} \in$ $\mathrm{Cn}(\mathrm{K})$ será relevante contar con información suficiente acerca de I (Balzer, Moulines \& Sneed 1987, pp. 86-92).

\footnotetext{
${ }^{9}$ En el núcleo teórico, junto a los modelos potenciales, actuales y parciales, están presentes también los conjuntos C y L. Por C se designa al conjunto de condiciones de ligadura (o constraints) a partir de las cuales se caracterizan las relaciones entre diferentes modelos de una misma teoría. Por $\mathbf{L}$ se designa al conjunto de los vínculos interteóricos (o links) a partir de los cuales se caracterizan las relaciones entre los elementos teóricos de una y otra teoría. En el presente trabajo se realizó una presentación incompleta de los componentes del núcleo teórico básico para la genética. Al respecto de condiciones de ligadura o constraints y vínculos interteóricos o links, ver Balzer, Moulines \& Sneed (1987, pp. 40-47, 57-62). Para el análisis de las condiciones de ligadura de la genética, puede consultarse Lorenzano (2002, pp. 279 280).
} 


\subsection{Los modelos de la genética molecular}

El elemento teórico básico para la genética molecular es presentado, de acuerdo con una reconstrucción estructuralista de la teoría, como un refinamiento de aquel propuesto para la genética. A fin de presentar el primero de los axiomas que permite definir los modelos para la genética molecular, algunas aclaraciones previas serán necesarias. Para comenzar, debe recordarse que si bien en los cromosomas de las células eucariotas la molécula de ADN se presenta generalmente en la forma lineal, algunas células procariotas presentan ADN circular. Dado que la forma más frecuente de ocurrencia de $\mathrm{ADN}$ es en forma lineal, en la reconstrucción de la teoría que aquí se ha tenido en cuenta se ha considerado únicamente esta posibilidad aplicando una noción general de strand (hebra). Esta noción es útil al momento de considerar al ADN como una entidad de forma prolongada, construida a partir de diferentes partes que pueden ser separadas. Así, esta entidad de forma prolongada puede ser conceptualizada como una secuencia de clases de objetos más pequeños. Las entidades de la forma de una secuencia serán llamadas strands, mientras que las entidades que conforman la secuencia se denominarán quanta (Balzer \& Dawe 1990, pp. 90-91). La molécula completa de ADN puede ser concebida como formando un strand, sin embargo, alternativamente, la propuesta de los autores en esta reconstrucción fué considerar sólo una de las cadenas o cordones de la doble hélice que constituyen la molécula como formando un strand. La razón más importante para ello es que la información genética está contenida en una (y otra) de las dos cadenas de la molécula de ADN y sólo una de las dos cadenas hace las veces de "molde" en el proceso de transcripción. La noción de un GENOTYPE propuesta en los modelos para la genética es presentada como especializada en el modelo para la genética molecular. De este modo, un GENOTYPE consistiría en un conjunto N de cadenas de nucleótidos, siendo cada cadena sólo una mitad de una molécula de ADN; y siendo cada miembro del conjunto correspondiente un cromosoma más una función posición $\psi$ que asigna posiciones espaciales a los nucleótidos. Así, los GENOTYPES son analizados como configuraciones de strands de la forma $\mathrm{C}=\left\langle\mathrm{N}, \mathbb{R}^{3}, \psi\right\rangle$ (Balzer \& Dawe 1990, pp. 45-46, 90).

En el caso de los quanta, la primera de las características que se intenta explicitar es que están ordenados en una cierta secuencia para formar los strands; conceptualmente, forman un "orden lineal" con respecto a alguna relación de orden $<$. Aquí, el orden lineal supondrá considerar, por un lado, al conjunto de los quanta $Q$ y, por otro lado, una relación binaria < sobre $Q$ que satisfaga los siguientes requisitos (Balzer \& Dawe 1990, p. 43):

1) Para todo $q, q^{\prime}, q^{\prime \prime} \in Q:$ si $q<q^{\prime}<q^{\prime \prime}$, entonces $q<q^{\prime \prime}$

2) Para todo $q \in$ Q: no $(q<q)$

3) Para todo $q, q^{\prime} \in Q: q<q^{\prime}$ o $q^{\prime}<q$

Debido a que la molécula de ADN se concibe como lineal y finita, la noción de strand es conceptualizada del siguiente modo:

$s$ es un strand si y sólo si $s$ consiste en un conjunto finito $Q$ y una relación binaria $<$ sobre $Q$ la cual es transitiva, anti-reflexiva y conectada. (Balzer \& Dawe 1990, p. 43)

En lo que respecta al $\mathrm{ADN}$, la relación < debe ser interpretada como expresando el ordenamiento de las ternas de bases a lo largo de la molécula de ADN, mientras que la secuencia completa de bases en una molécula (o una parte de una secuencia tal) es aquello a lo que se hace referencia con el término strand (Balzer \& Dawe 1990, pp. 43-44). Los quanta, por otro lado, son interpretados como codones o ternas de bases. Un quantum estará representando una secuencia de tres de las cuatro bases: Adenina (A), Guanina (G), Timina (T) y Citosina (C) con uniones fosfodiéster entre cualesquiera dos sucesivas. La segunda característica que debe ser explicitada acerca del ordenamiento de los quanta es que, en la definición de los strands, este ordenamiento pueda ser definido haciendo referencia a nociones químicas. Es decir, para dos quanta $\mathrm{B}, \mathrm{B}^{\prime}$ en $\mathrm{Q}$, entonces $\mathrm{B} \prec \mathrm{B}^{\prime}$ si y sólo si están unidas mediante un 
puente fosfodiéster tal que el grupo 3'-OH de B se encuentra con el grupo 5'-OH de B'. A partir de esto, en la reconstrucción es posible definir el concepto de ordenamiento químico natural del siguiente modo: dos quanta $\mathrm{B}, \mathrm{B}^{\prime}$ en $\mathrm{Q}$ se encuentran en una relación de orden $<$ (es decir, $\mathrm{B}<\mathrm{B}^{\prime}$ ) si y sólo si existen $B, B^{\prime}$ en $Q$ tal que $B=B_{1} \prec \ldots \prec B_{n}=B^{\prime}($ Balzer $\&$ Dawe 1990, p. 95).

A partir de lo anterior, entonces, el primero de los axiomas para la genética molecular expresaría lo siguiente (Balzer \& Dawe 1990, p. 95):

AGM1. Existe un número $k$ tal que todos los GENOTYPES $\gamma$ son configuraciones de strands de la forma $\left\langle N, \mathbb{R}^{3}, \psi\right\rangle$ tales que:

1) $N$ tiene exactamente $k$ elementos $\left(N=\left\{s_{1}, s_{k}\right\}\right)$

2) Para todo $i \leq k$ : si strand $s_{\mathrm{i}}$ en $N$ tiene la forma $\left\langle Q_{\mathrm{i}},<_{\mathrm{i}}\right\rangle$ entonces

2.1) cada elemento de $Q_{i}$ es una terna

2.2) $<_{i}$ es un ordenamiento químico natural sobre $Q_{i}$

Para la formulación de los siguientes dos axiomas de la genética molecular se asume que un PHENOTYPE consiste en un conjunto de secuencias ordenadas de aminoácidos. Cada secuencia en un PHENOTYPE corresponde a un strand de un GENOTYPE, y los conjuntos de secuencias y strands son mapeados uno a uno, uno tras otro. Por lo tanto, el conjunto de secuencias que constituyen un PHENOTYPE no es sino un conjunto de strands. De ahí que se distinga entre strandp y strand $d_{\mathrm{G}}$ para indicar secuencias ordenadas correspondientes a un PHENOTYPE y a un GENOTYPE, respectivamente. Teniendo presente la definición de strand presentada anteriormente, los quanta de un $\operatorname{strand}_{\mathrm{P}}$, es decir, los elementos a partir de los cuales son formadas las secuencias serán aquí los aminoácidos. El ordenamiento químico entre estos será representado por el símbolo “o ”. Un strandp, entonces, es una estructura $\left\langle\mathrm{A},{ }^{\circ}\right\rangle$ donde $\mathrm{A}$ denota el conjunto de aminoácidos y ${ }^{\circ}$ su ordenamiento químico a lo largo de la secuencia. Así, el segundo axioma afirma (Balzer \& Dawe 1990, p. 96):

AGM2. Existe un número $r$ tal que cada PHENOTYPE es un conjunto de $r$ strandsp de aminoácidos, y $r$ es idéntico al número $k$ de strands $s_{\mathrm{G}}$ en los GENOTYPES.

Para presentar el siguiente axioma de la genética molecular, se introduce una nueva función "EX" (expresión) que mapea codones a aminoácidos. La función EX será tratada como una función definida sobre el conjunto de todas las ternas (TRIPLET) en el conjunto de todos los aminoácidos (A-ACID). Así, EX: TRIPLET $\rightarrow$ A-ACID (Balzer \& Dawe 1990, pp. 97-98).

AGM3.

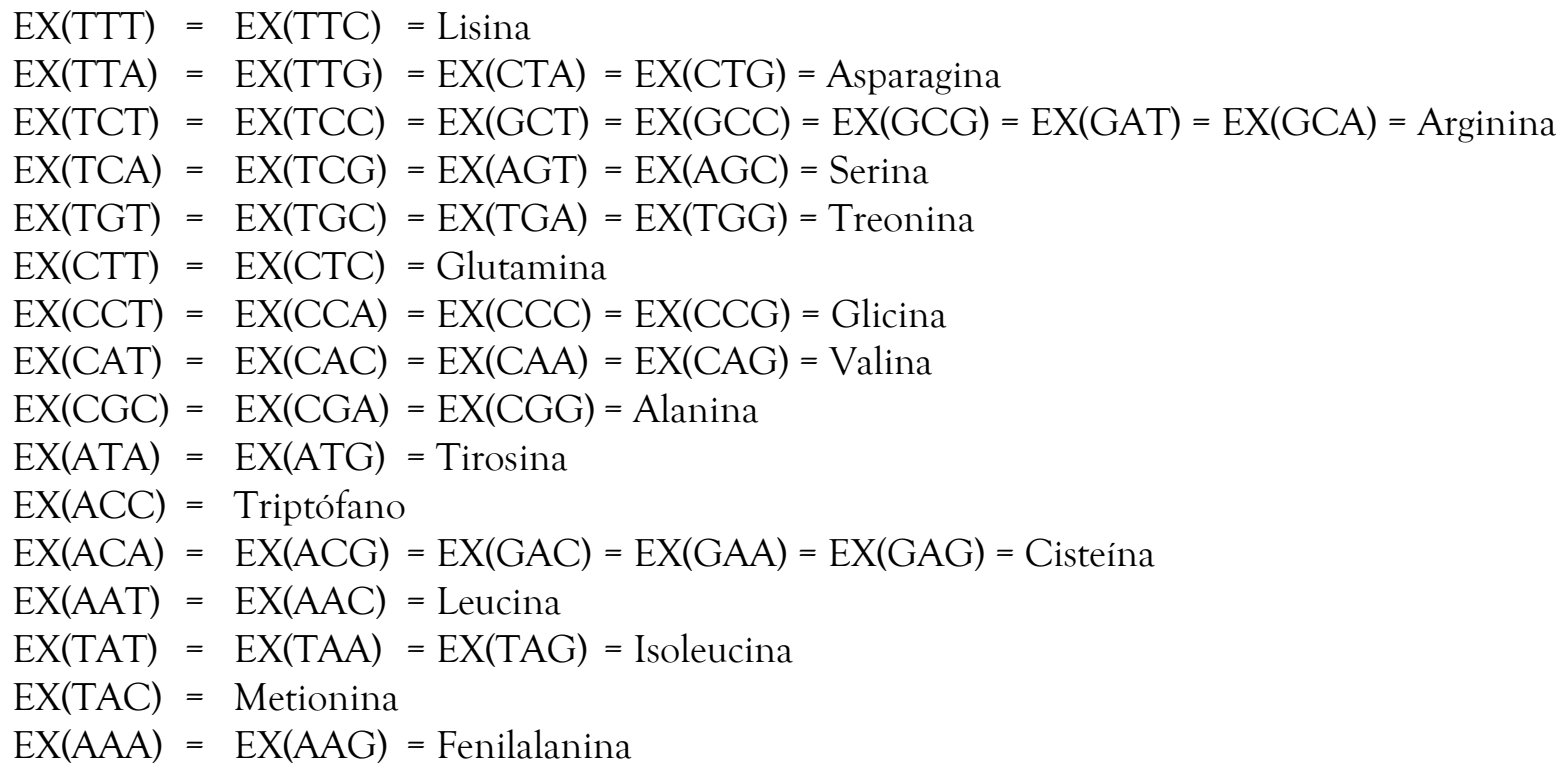




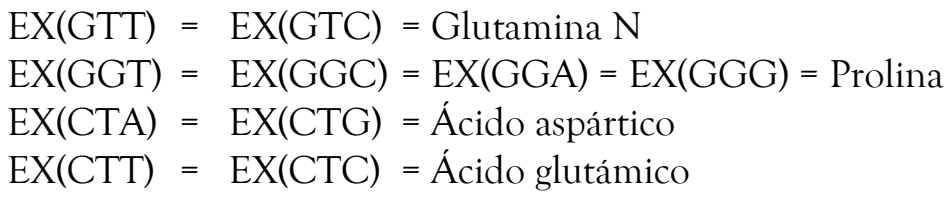

En la caracterización de los modelos para la genética se presentó la función DETERMINER la cual permitía asignar PHENOTYPES a GENOTYPES. Aquí se introducirá una nueva función COR ("correlación") que permite caracterizar con mayor detalle a la función DETERMINER. A fin de lograr esto, COR se introduce como una función definida sobre el conjunto de todos los strands presentes en los diferentes GENOTYPES de un modelo. A tal conjunto se lo llamará STRAND(G). Por otro lado, se llamará STRAND(P) al conjunto de todos los strandsp presentes en los PHENOTYPES de un modelo. Así, COR: STRAND(G) $\rightarrow$ STRAND(P). A partir de esta función, entonces, el cuarto axioma de la genética molecular puede formularse de la siguiente manera (Balzer \& Dawe 1990, p. 99):

AGM4. Si P y G son los conjuntos de PHENOTYPES y GENOTYPES respectivamente, entonces existen funciones

COR: STRAND $(G) \rightarrow$ STRAND(P) y EX: TRIPLET $\rightarrow$ A-ACID.

Tal que para todo PHENOTYPE $\pi$ en $\mathrm{P}$ y todo GENOTYPE ${ }^{\circ}$ en $\mathrm{G}$ se cumple lo siguiente:

Si $\pi$ tiene la forma $\left\{s_{1}^{*}, \ldots, s_{k}^{*}\right\}$ y $\gamma$ tiene la forma $\left\langle N, \mathbb{R}^{3}, \psi\right\rangle$ con $N=\left\{s_{1}, s_{k}\right\}$, entonces: $\operatorname{DETERMINER}\left({ }^{\circ}\right)=\pi$ syss

1) COR, restringida a $N$, sobre $\left\{s_{1}^{*}, \ldots, s_{k}^{*}\right\}$

2) Para todo $i, j \leq k$, si $\operatorname{COR}\left(s_{i}\right)=s_{j}^{*}$ y $s_{i}, s_{j}^{*}$ tienen las formas $s_{i}=\left\langle Q_{i},<_{i}\right\rangle, s_{j}^{*}=\left\langle Q_{j}^{*}, \prec_{j}\right\rangle$, respectivamente, entonces

2.1) EX, restringida a $Q_{i}$, sobre $Q_{j}^{*}$

2.2) EX, restringida a $Q_{i}$, conserva el orden, es decir, para todo $q, q^{\prime} \in Q_{i}: q<q_{i}^{\prime}$ syss $\operatorname{EX}(q) \circ \operatorname{EX}\left(q^{\prime}\right)$

En este caso, la única función que aparece es COMBINATOR. Como se indicó antes, para el caso de la genética, esta función representa la transición de genotipos paternos a genotipos en la descendencia. Para el caso de la genética molecular fue asumido que si $\gamma \in$ GENOTYPE, entonces sería considerado como una configuración $\mathrm{C}=\left\langle\mathrm{N}, \mathbb{R}^{3}, \psi\right\rangle$ de strands. Usando, entonces, las variables $\mathrm{C}, \mathrm{C}^{\prime}, \mathrm{C}_{i}$ (en lugar de $\left.\gamma, \gamma, \gamma_{1}\right)$, puede expresarse la conexión establecida por COMBINATOR entre los genotipos parentales y los de la descendencia de la siguiente manera: COMBINATOR(C, C') $=\sum \alpha_{i} \mathrm{C}_{i}$

Esto permitiría considerar un modelo de transición cinemática a partir del cual pudieran describirse todas las maneras en que los strands parentales se combinan para dar forma a los strands de la progenie. Un modelo tal puede ser presentado haciendo posible formular distintos tipos de combinaciones cinemáticas. ${ }^{10}$ A partir de esto último, el axioma para la genética molecular afirma (Balzer \& Dawe 1990, p. 101):

AGM5. Para todos los GENOTYPES $\gamma, \gamma, \gamma_{1}, \ldots, \gamma_{n}$ y todos los $\alpha_{1}, \ldots, \alpha_{n}$ : si $\operatorname{COMBINATOR}(\gamma, \gamma)=$ $\sum \alpha_{i} \gamma_{i}$, entonces para cada $i \leq n: \gamma_{i}$ puede ser obtenida a partir de $\gamma$ y $\gamma$ por medio de una combinación cinemática.

Los modelos definidos a partir de este último axioma, entonces, intentan capturar el fenómeno de combinación y recombinación de las cadenas de ADN durante los procesos de meiosis y fertilización.

\footnotetext{
${ }^{10}$ Las precisiones acerca de estos tipos de combinaciones se hacen posibles a partir de los modelos de transición cinemática. Ver Balzer \& Dawe (1990, cap. 3).
} 
Por otra parte, los axiomas 2, 3 y 4 determinan modelos a partir de los cuales pueda representarse el proceso de trascripción genética de ADN a secuencias de aminoácidos. Y, finalmente, lo concerniente a la estructura de la molécula de $\mathrm{ADN}$ queda expresado a partir del axioma 1 para la genética molecular.

A diferencia de lo que ocurría al considerar el modelo icónico de doble hélice, es decir, la maqueta de alambre y cartón de Watson, a partir de una propuesta de reconstrucción en el marco de la metateoría estructuralista lo que se presenta es un modelo teórico o formal de doble hélice. En este caso, expresado en el lenguaje de la teoría de conjuntos.

Como se ha visto, partiendo de la reconstrucción de la genética molecular, el modelo de doble hélice se constituye en uno de sus modelos. Específicamente, a partir del primer axioma para la genética molecular, aquel que permite presentar el modelo de doble hélice, se establece cuáles son y qué estructura poseen aquellas entidades respecto de las cuales podrían valer los últimos dos axiomas.

En lo que respecta a la aserción empírica, Watson y Crick estarían afirmando que la estructura de toda molécula de ADN consiste idealmente en dos cadenas (en el modelo, una configuración $\mathrm{C}=\langle\mathrm{N}$, $\left.\mathbb{R}^{3}, \psi\right\rangle$ de strands con $N=\left\{s_{1}, s_{k}\right\}$ ), en las que se encuentran bases nitrogenadas especialmente ordenadas (en el modelo, $s_{\mathrm{i}}$ en $N$ tiene la forma $\left\langle\mathrm{Q}_{\mathrm{i}},<_{\mathrm{i}}\right\rangle$, donde $\mathrm{Q}_{\mathrm{i}}$ es un codón $\mathrm{y}<_{\mathrm{i}}$ es un ordenamiento químico natural sobre $Q_{\mathrm{i}}$ ).

\section{Consideraciones finales}

De acuerdo con el realismo constructivo de Ronald Giere, las idealizaciones presentadas en el artículo de Watson y Crick constituirían las hipótesis a partir de las cuales se hace posible definir un modelo teórico acerca de la estructura del ADN. Estas hipótesis, sin embargo, pudieron precisarse a partir de la construcción de modelos a escala de la molécula. Para Giere, tanto el modelo teórico (las hipótesis) como el modelo a escala o icónico (la maqueta de alambre y cartón) tienen la función de representar un sistema real concreto, a saber, cualquier porción de una molécula de ADN. La hipótesis teórica afirma que cualquier porción de una molécula de ADN es similar en ciertos aspectos y grados a la entidad abstracta presentada por el modelo teórico. Los modelos teóricos son intentos de representar de un modo abstracto los sistemas reales, ofrecen una idealización; mientras que los modelos icónicos constituyen intentos concretos a partir de los cuales puedan ser capturadas algunas, pero no necesariamente todas, las propiedades de las entidades a las cuales representan. De este modo, para Giere, la importancia de los modelos icónicos radica en que resultan medios útiles para alcanzar o desarrollar modelos teóricos.

En cuanto al análisis estructuralista del modelo de doble hélice, lo expuesto constituiría la formulación en lenguaje conjuntista del modelo teórico para la estructura de la molécula de ADN. Tal modelo se presenta formando parte de la clase de modelos a partir de los cuales se identifica a la genética molecular, la cual a su vez puede defenderse como un refinamiento de la genética. La relación entre este modelo formal o abstracto de doble hélice con aquella entidad empírica a la cual desea capturar se conceptualizó como la relación entre el núcleo teórico básico de la genética molecular y una de sus aplicaciones intencionales. Tal relación es expresada por la aserción empírica. Resumidamente, la aserción empírica afirmaba que la estructura constituida a partir de la descripción de cualquier porción de una molécula de ADN pertenece a, o es un subconjunto de, los modelos parciales de la genética. La estructura constituida a partir de la descripción de cualquier porción de una molécula de ADN es un modelo parcial que además satisface las leyes y demás restricciones impuestas desde la teoría. A partir de esto, debe reconocerse que la tarea de acercar la teoría y la experiencia, es decir, entidades abstractas y sistemas reales, resulta muy compleja, más aun si el modelo teórico alcanza un alto grado de formalización. Para el estructuralismo, desde la teoría, los modelos potenciales parciales, aun siendo entidades abstractas, se ofrecen, por así decirlo, acortando la distancia entre la teoría y los sistemas empíricos que la teoría quiere capturar. 
Astbury, W. T. (1947), "X-Ray Studies of Nucleic Acid”, Symposia of the Society for Experimental Biology 1: 66-76.

Balzer W. (1997), Teorias empiricas: modelos, estructuras y ejemplos, Madrid: Alianza. (Versión castellana revisada de: Empirische Theorien: Modelle-Strukturen-Beispiele, Braunschweig: Vieweg, 1982.)

Balzer W. y C. M. Dawe (1990), Models for Genetics, München: Institut für Philosophie, Logik und Wissenschaftstheorie.

Balzer, W., Moulines, C. U. y J. D. Sneed (1987), An Architectonic for Science. The Structuralist Program, Dordrecht: Reidel. (Versión castellana de Pablo Lorenzano: Una arquitectónica para la ciencia. El programa estructuralista, Bernal: Universidad Nacional de Quilmes, 2012.)

Díez, J. A. y P. Lorenzano (eds.)(2002), Desarrollos actuales de la metateoría estructuralista: problemas y discusiones, Quilmes: Universidad Nacional de Quilmes/Universidad Autónoma de Zacatecas/Universidad Rovira i Virgili.

Díez, J. A. y P. Lorenzano (2002a), "La concepción estructuralista en el contexto de la filosofía de la ciencia del siglo XX”, en Díez \& Lorenzano (2002), pp. 13-78.

Franklin, R. E. y R. G. Gosling (1953), “Molecular Configuration in Sodium Thymonucleate”, Nature 171: 740-741.

Giere, R. N. (1979), Understanding Scientific Reasoning, New York: Holt Rinehart \& Winston, $3^{\text {a }}$ ed. revisada, 1991

Giere, R. N. (1985), “Constructive Realism”, en Churchland, P. y C. Hooker (eds.), Images of Science, Chicago: University of Chicago Press, pp. 75-98.

Giere, R. N. (1988), Explaining Science: A Cognitive Approach, Chicago: University of Chicago Press.

Giere, R. N. (1994), “The Cognitive Structure of Scientific Theories”, Philosophy of Science 61: 276-296.

Giere, R. N. (1999), “Using Models to Represent Reality”, en Magnani, L., Nersessian, N. J. y P. Thagard (eds.), ModelBased Reasoning in Scientific Discovery, New York: Kluwer/Plenum, pp. 41-57.

Giere, R. N. (2004), "How Models are Used to Represent Reality”, Philosophy of Science 71(5): 742-752.

Lorenzano, P. (2002), “La teoría del gen y la red teórica de la genética”, en Díez \& Lorenzano (2002), pp. 263-303.

Lorenzano, P. (2005), “Ejemplares, modelos y principios en la genética clásica”, Scientiae Studia 3(2): 185-203.

Pauling, L. C. y R. B. Corey (1953a), "Structure of the Nucleic Acids”, Nature, 171: 346.

Pauling, L. C. y R. B. Corey (1953b), “A Proposed Structure for the Nucleic Acids”, Proceedings of the National Academy of Sciences U.S.A. 39: 84-97.

Suppe, F. (1977), The Structure of Scientific Theories, Urbana, IL: University of Illinois Press.

Suppes, P. (1960), "A Comparison of the Meaning and Uses of Models in Mathematics and the Empirical Sciences", Synthese 12: 287-301. (Versión castellana: "Una comparación del significado y los usos de los modelos en las matemáticas y las ciencias empíricas”, en Estudios de filosofía y metodología de la ciencia, Madrid: Alianza, 1988, pp. 109-123.)

Suppes, P. (1981), Introducción a la lógica simbólica, México: CECSA.

Watson, J. D. y F. H. C. Crick (1953a), "Molecular Structure of Nucleic Acid: A Structure for Deoxyribose Nucleic Acid", Nature 171: 737-738.

Watson, J.D. y F.H.C. Crick (1953b), "Genetical Implications of the Structure of Deoxyribonucleic Acid”, Nature 171: 964-967.

Wilkins, M.H.F. y J.T. Randall (1953), "Cristallinity in Sperm Heads. Molecular Structure of Nucleoprotein in vivo", Biochimica et Biophysica Acta 10: 192-193.

Wyatt, G.R. (1952), “The Nucleic Acids of some Insect Viruses”, The Journal of General Physiology 36: 201-205. 
30 | María de las Mercedes O’Lery

Zamenhof, S., Brawerman, G. y E. Chargaff (1952), "On the Desoxypentose Nucleic Acids from Several Physical and Chemical Agents”, Biochimica et Biophysica Acta 9: 402-405. 